\title{
References
}

1 Hatano S, Strasser T. Primary Pulmonary Hypertension: Report on a WHO Meeting, Geneva, 15-17 October 1973. Geneva, World Health Organization, 1975.

2 Galiè N, Hoeper MM, Humbert M, et al. Guidelines for the diagnosis and treatment of pulmonary hypertension. Eur Respir J 2009; 34: 1219-1263.

3 Kovacs G, Berghold A, Scheidl S, et al. Pulmonary arterial pressure during rest and exercise in healthy subjects: a systematic review. Eur Respir J 2009; 34: 888-894.

4 Kovacs G, Olschewski A, Berghold A, et al. Pulmonary vascular resistances during exercise in normal subjects: a systematic review. Eur Respir J 2012; 39: 319-328.

5 Tolle JJ, Waxman AB, Van Horn TL, et al. Exercise-induced pulmonary arterial hypertension. Circulation 2008; 118: $2183-2189$

6 Lewis GD, Murphy RM, Shah RV, et al. Pulmonary vascular response patterns during exercise in left ventricular systolic dysfunction predict exercise capacity and outcomes. Circ Heart Fail 2011; 4: 276-285.

7 Herve P, Lau EM, Sitbon O, et al. Criteria for diagnosis of exercise pulmonary hypertension. Eur Respir J 2015; 46: $728-737$.

8 Condliffe R, Kiely DG, Peacock AJ, et al. Connective tissue disease-associated pulmonary arterial hypertension in the modern treatment era. Am J Respir Crit Care Med 2009; 179: 151-157.

9 Naeije R, Vanderpool R, Dhakal BP, et al. Exercise-induced pulmonary hypertension: physiological basis and methodological concerns. Am J Respir Crit Care Med 2013; 187: 576-583.

10 Butler J, Schrijen F, Henriquez A, et al. Cause of the raised wedge pressure on exercise in chronic obstructive pulmonary disease. Am Rev Respir Dis 1988; 138: 350-354.

\section{Tuberculosis in migrants from 106 countries to Italy, 2008-2014}

\section{To the Editor:}

Tuberculosis (TB) is a major infectious disease worldwide. Over recent years, TB caused by multidrug-resistant (MDR) Mycobacterium tuberculosis strains (resistant to at least isoniazid and rifampicin) and extensively drug-resistant (XDR) strains (MDR strains resistant to any fluoroquinolone and to at least one injectable second-line drug (SLD), i.e. kanamycin, capreomycin or amikacin) has emerged as a public health concern in industrialised countries, due to increasing migration from regions where TB is endemic.

In Italy, while the number of TB cases decreased from 4220 in 2004 to 3153 in 2013 (7.3 and 5.3 cases per 100000 population, respectively) [1, 2], notifications from foreign-born persons (FBPs) increased from 39.4\% in 2004 to $63 \%$ in 2013. The association of MDR-TB with immigrant status was previously reported [3, 4] but a systematic study of the country of origin of migrants was not performed. The European Centre for Disease Prevention and Control (ECDC) published a report on key infectious diseases (including TB) affecting migrant populations in the European Union/European Economic Area for the years 2000-2010 [5]. Here, we conducted a retrospective analysis in Italy over the period 2008-2014 in order to estimate TB burden and MDR/XDR-TB in FBPs by country of origin, in comparison with Italian-born persons (IBPs), and to determine resistance to SLDs in MDR/XDR M. tuberculosis clinical isolates.

Our laboratory network, the Italian Multicentre Study on Resistance to Antituberculosis drugs (SMIRA), is composed of 35 hospital reference laboratories located in 18 out of 20 regions. All the laboratories are periodically examined by first-line drug (FLD) and SLD proficiency testing exercises by the World Health Organization (WHO) Supranational Reference Laboratory in Rome [6]. In 2013, the SMIRA network covered $74.5 \%$ of nationwide notified cases, contributing the majority of cases included in the annual ECDC/WHO TB report [2].

TB cases with positive M. tuberculosis cultures were routinely examined by the SMIRA laboratories for susceptibility to FLD and SLD. Table 1 shows data on drug resistance of $13030 \mathrm{M}$. tuberculosis strains with known country of origin (6869 from FBPs and 6161 from IBPs) isolated from 13030 different patients in 2008-2014. Due to difficulties in obtaining a reliable history of prior treatment in FBPs, we were not able to distinguish new cases from previously treated cases, thus the number of TB cases included both categories. MDR-TB cases decreased from 3.7\% in 2008 to $3.1 \%$ in 2014.

M. tuberculosis strains were isolated from migrants coming from 106 countries, including patients with MDR strains from 37 countries and with non-MDR strains from 69 countries (table 1). Data of MDR strains from 
TABLE 1 Geographic distribution of 13030 Mycobacterium tuberculosis strains isolated in Italy in 2008-2014 from 6869 foreign-born persons (FBPs) and 6161 Italian-born persons (IBPs) with tuberculosis

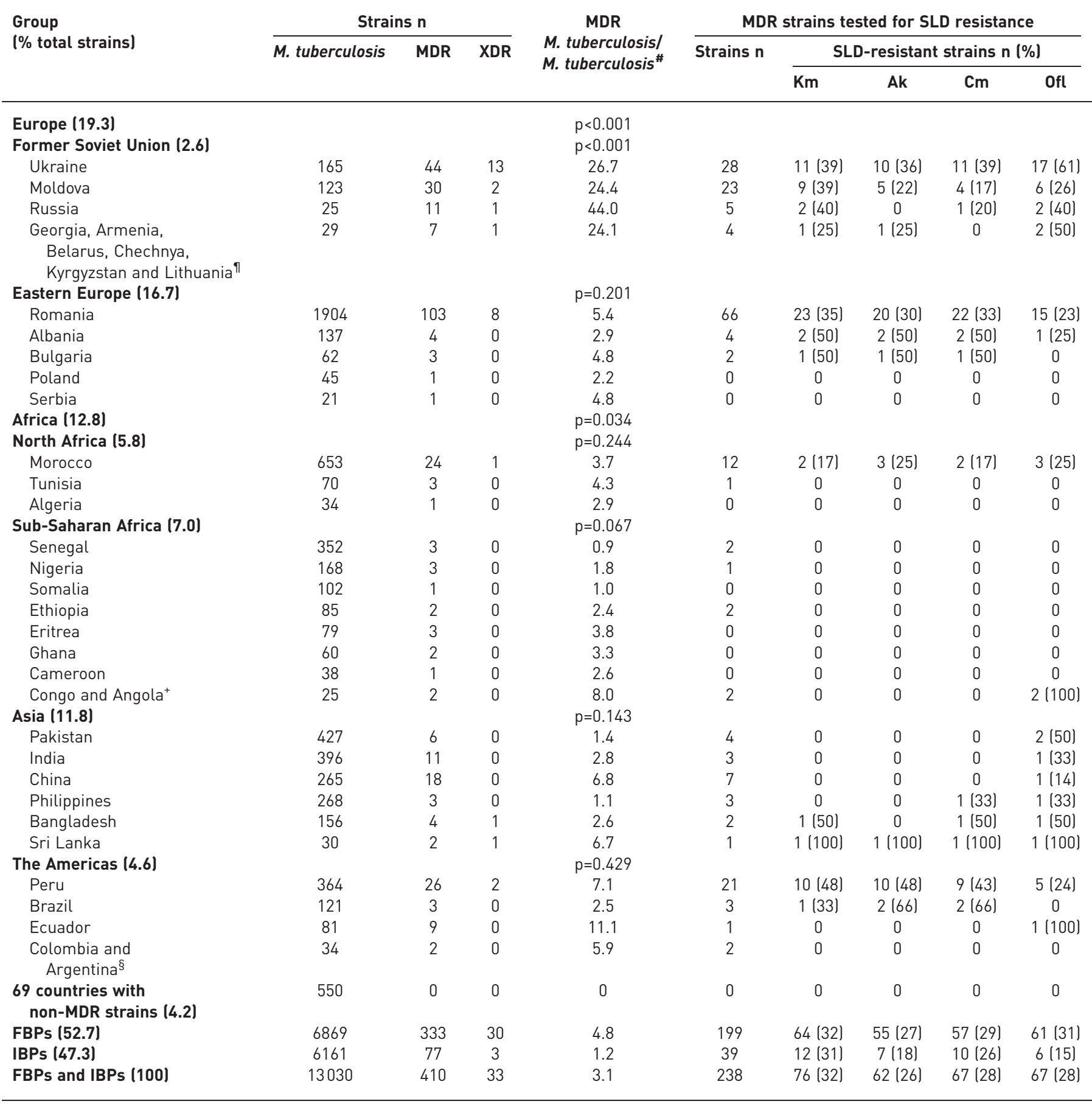

FBP strains were isolated from people coming from 106 countries, including patients with multidrug-resistant (MDR) strains from 37 countries and with non-MDR strains from 69 countries. XDR: extensively drug-resistant. SLD: second-line drug; Km: kanamycin; Ak: amikacin; Cm: capreomycin; Ofl: ofloxacin. " : p-values (Student's t-test) for percentages of MDR M. tuberculosis/M. tuberculosis of the countries of a continent/ region (in bold) versus percentages of MDR M. tuberculosis/M. tuberculosis of all other countries, excluding Italy. ": MDR M. tuberculosis/ M. tuberculosis: Georgia 2/22; Armenia 1/1; Belarus 1/2; Chechnya 1/1; Kyrgyzstan 1/1; Lithuania 1/2. ${ }^{+}$: MDR M. tuberculosis/M. tuberculosis: Congo 1/20; Angola 1/5. §;: MDR M. tuberculosis/M. tuberculosis: Colombia 1/24; Argentina 1/10.

FBPs (333 out of 410) were stratified by continent (Europe, Africa, Asia and the Americas) and country of origin. Due to known differences in MDR-TB rates in European and African countries [7, 8], Europe (14 countries) was subdivided in the former Soviet Union and Eastern Europe regions, and Africa 
(12 countries) into North Africa and sub-Saharan regions. Migrants also came from six Asian countries and five countries in the Americas (all in South America).

Large differences were found in MDR-TB rates (MDR M. tuberculosis/M. tuberculosis strains), with the ranking (from greatest to least) being: former Soviet Union (mean \pm SD 29.8 $\pm 9.5 \%$, range 24.1-44.0\%); the

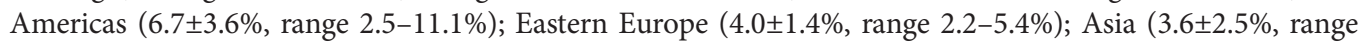

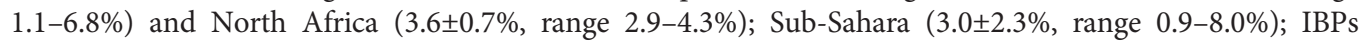
(1.2\%). The differences between MDR-TB percentages of the countries of a continent/region versus MDR-TB percentages of all other countries (excluding Italy) were statistically significant $(\mathrm{p}<0.05$ by Student's t-test $)$ for Europe $(p<0.001)$, the former Soviet Union $(p<0.001)$ and Africa $(p=0.034)$. The sub-Saharan region showed a $\mathrm{p}$-value of 0.067 , close to cut-off for significance. No significant differences were seen for Eastern Europe, North Africa, Asia or the Americas.

The highest MDR-TB rates were observed in FBPs from the former Soviet Union (Ukraine, Moldova, Russia, Georgia, Armenia, Belarus, Chechnya, Kyrgyzstan and Lithuania), in accordance with the fact that these countries, with the exception of Chechnya, belong to the WHO list of 27 high MDR-TB burden countries [7]. Indeed, MDR-TB levels were consistent with those of their native countries (e.g. in 2013: Ukraine, 26.0\%; Moldova, 33.8\%; Russia, 28.5\%; Georgia, 16.6\%; Armenia, 15.1\%; Belarus, 39.7\%; Kyrgyzstan, 36.8\%; Lithuania, 19.2\%) [2]. Among the Eastern European countries, Romania was the largest TB group and showed the highest MDR-TB rate (5.4\%). As for Asia and the Americas, the highest rates were observed in migrants from China (6.8\%) and Ecuador (11.1\%), respectively.

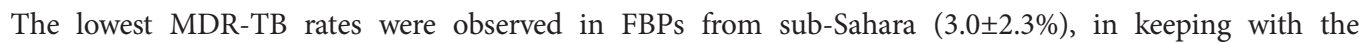
knowledge that MDR-TB in new cases in African countries is <3\% [7]. Values ranged from $0.9 \%$ (Senegal) to $8 \%$ (Congo and Angola, pooled for low case numbers). Nigeria and Ethiopia, also belonging to the 27 WHO countries group [7], showed $1.8 \%$ and $2.4 \%$ MDR-TB, respectively. MDR-TB from North Africa was $2.9-4.3 \%$. A recent meta-analysis [8] reporting low MDR-TB in new cases and previously treated cases in 21 sub-Saharan countries (including Ethiopia, Somalia, Ghana and Cameroon) is in agreement with our observations. Some investigators also found low levels of MDR-TB in Sub-Sahara/Africa [9, 10] while others reported increasing MDR-TB in five African countries other than those of table 1 [11]. Low MDR-TB rates in sub-Sahara/Africa were ascribed to several factors including poor diagnostic and surveillance [9], wide use of fixed-dose combinations [8], and the fact that for many years Africa was neglected and TB was not treated [10].

The ranking of XDR-TB rates (XDR $M$. tuberculosis strains/M. tuberculosis strains) in $33 \mathrm{XDR}$ strains isolated from 10 countries (including Italy) was as follows: former Soviet Union (17 strains, 4.97\%); Eastern Europe (eight strains, 0.37\%); the Americas (two strains, 0.33\%); Asia (two strains, 0.13\%) and North Africa (one strains, 0.13\%); IBPs (three strains, 0.05\%); sub-Sahara (no strains, 0\%); reflecting the MDR-TB trend.

Among 238 MDR strains tested for susceptibility to SLDs (199 from FBPs and 39 from IBPs), resistance in FBPs and IBPs was similar for kanamycin (32\% and 31\%, respectively) and capreomycin (29 and $26 \%$, respectively), while it was higher in FBPs than in IBPs for amikacin (27\% and $18 \%$, respectively) and ofloxacin ( $31 \%$ and $15 \%$, respectively), which is likely to be due to the large use of amikacin and fluoroquinolones for treatment of MDR-TB in FBPs. Indeed, resistance to fluoroquinolones and other drugs is increasing in high TB burden countries with weak healthcare systems [12, 13]. Only some of the MDR strains (238 out of 410) were tested for SLD resistance because in the SMIRA network, the first SLD proficiency testing was performed in 2010 [6], thus only MDR strains isolated in 2011-2014 were considered.

Overall, a consistent migration of TB patients from 106 countries occurred in Italy in 2008-2014, with MDR- and XDR-TB being very high in FBPs from the former Soviet Union and low from sub-Sahara. Our data show that the MDR-TB rates in migrants reflects those in the countries of origin. These findings should guide clinicians in their approach to TB in migrants, using all available diagnostic tools to rapidly start with treatment of the populations at high risk of MDR/XDR-TB. In addition, monitoring drug resistance rate in migrants in low-incidence countries can provide information on the rate in the countries of origin, in the framework of the WHO vision to eliminate TB as a public health problem by $2035[14,15]$.

@ERSpublications

In migrants coming to Italy from 106 countries, MDR-TB was high from the former Soviet Union and low from Africa http://ow.ly/WZDbo

Lanfranco Fattorini ${ }^{1}$, Alessandro Mustazzolu ${ }^{1}$, Emanuele Borroni ${ }^{2}$, Giovanni Piccaro ${ }^{1}$, Federico Giannoni ${ }^{1}$, Daniela Maria Cirillo ${ }^{2}$ and the Italian Multicentre Study on Resistance to Antituberculosis Drugs (SMIRA) Group ${ }^{3}$ ${ }^{1}$ Dipartimento di Malattie Infettive, Parassitarie e Immunomediate, Istituto Superiore di Sanità, Rome, Italy. ${ }^{2}$ Emerging Bacterial Pathogens Unit, San Raffaele Scientific Institute, Milan, Italy. ${ }^{3}$ For a full list of the SMIRA group members and their affiliations see the Acknowledgements section. 
Correspondence: Lanfranco Fattorini, Dipartimento di Malattie Infettive, Parassitarie e Immunomediate, Istituto Superiore di Sanità, Viale Regina Elena 299, 00161 Rome, Italy. E-mail: lanfranco.fattorini@iss.it

Received: Nov 062015 | Accepted after revision: Dec 152015 | First published online: Feb 042016

Support statement: This study was supported by the CCM Project of the Italian Ministry of Health. Funding information for this article has been deposited with FundRef.

Conflict of Interest: None declared.

Acknowledgements: The members of the SMIRA laboratory network are V. Ghisetti, A. Mondo and R. Milano (Ospedale A. di Savoia, Turin, Italy), A. Barbui (Ospedale Molinette, Turin), P. Lorenzetti (Ospedale di Aosta, Aosta, Italy), G. De Renzi and M.G. Chirillo (Ospedale di Orbassano, Orbassano, Italy), G. Molinari and A. Camaggi (AOU Maggiore della Carità, Novara, Italy), P. Gritti, O. Varnier and E. Senno (Ospedale S. Martino, Genoa, Italy), E. Mazzola and G. Gesu (Ospedale Niguarda, Milan, Italy), P. Cichero (Ospedale S. Raffaele, Milan), A. Lombardi (Ospedale Sacco, Milan), E. Libanori and S. De Lorenzo (Ospedale di Sondalo, Sondalo, Italy), G. Pinsi (Spedali Civili, Brescia, Italy), P. Marone and V. Monzillo (Fondazione IRCCS S. Matteo, Pavia, Italy), C. Farina and M. Arosio (Ospedale Papa Giovanni XXIII, Bergamo, Italy), M. Peracchi and R. Manganelli (Ospedale di Padova, Padua, Italy), C. Fabris (Ospedale di Trieste, Trieste, Italy), C. Scarparo (AOU di Udine, Udine, Italy), C. Pedrotti and I. Caola (Ospedale di Trento, Trent, Italy), E. Frizzera and C. Larcher (Ospedale di Bolzano, Bolzano, Italy), P. Dal Monte and G. Lombardi (Policlinico S. Orsola-Malpighi, Bologna, Italy), A. Fabio and A. la Regina (Policlinico di Modena, Modena, Italy), M. Matteucci (Area Vasta di Romagna, Cesena, Italy), C. Piersimoni (Ospedali Riuniti, Ancona, Italy), M.T. Simonetti and E. Tortoli, (Ospedale Careggi, Florence, Italy), L. Rindi (Università di Pisa, Pisa, Italy), R. Mazzolla and E. Luciano (Ospedale di Perugia, Perugia, Italy), I. Sanguigni, P. Chiaradonna, M. Tronci, G. Parisi, S. Natili and A.M. Altieri (Ospedale S. Camillo-Forlanini, Rome, Italy), E. Bordi, P. De Mori and S. D’Arezzo (Ospedale L. Spallanzani, Rome), M. Sali, G. Delogu and M. Sanguinetti (Policlinico A. Gemelli, Rome), C. Russo and L. Coltella (Ospedale Bambino Gesù, Rome), M. Meledandri and M. Ballardini (Ospedale S. Filippo Neri, Rome), G. Tomei (Ospedale di Chieti, Chieti, Italy), G. Santoro and M. Conte (Ospedali dei Colli, Naples, Italy), N. Nuzzolese, C. Colonna, L. Buono, A. Sinno and E. Vitullo (Ospedale di Matera, Matera, Italy), D. Costa (Policlinico di Bari, Bari, Italy). A. Grimaldi (Ospedale di Triggiano, Bari), A. Di Taranto and R. De Nittis (AOU, OORR, Foggia, Italy), S. Dodaro, P. Cavalcanti and C. Giraldi (AO di Cosenza, Cosenza, Italy), S. Nisticò (Ospedale di Lamezia Terme, Lamezia Terme, Italy), C. Di Naso (Ospedale Garibaldi Centro, Catania, Italy), and R. Caddeu (Ospedale Binaghi, Cagliari, Italy).

\section{References}

1 European Centre for Disease Prevention and Control. Surveillance of Tuberculosis in Europe: EuroTB - Report on Tuberculosis Cases Notified in 2004. http://ecdc.europa.eu/en/publications/Publications/SUR_TB_EuroTB_ Annual_report_2004_full\%20report.pdf Date last updated: March 1, 2006.

2 European Centre for Disease Prevention and Control. Surveillance Report: Tuberculosis Surveillance and Monitoring in Europe - 2015. http://ecdc.europa.eu/en/publications/Publications/tuberculosis-surveillance-monitoring-Europe2015.pdf Date last updated: March 17, 2015.

3 Fattorini L, Mustazzolu A, Piccaro G, et al. Drug-resistant tuberculosis among foreign-born persons in Italy. Eur Respir J 2012; 40: 497-500.

4 Ingrosso L, Vescio F, Giuliani M, et al. Risk factors for tuberculosis in foreign-born people (FBP) in Italy: a systematic review and meta-analysis. PLoS One 2014; 9: e94728.

5 European Centre for Disease Prevention and Control. Technical Report: Assessing the Burden of Key Infectious Diseases Affecting Migrant Populations in the EU/EEA. http://ecdc.europa.eu/en/publications/Publications/ assessing-burden-disease-migrant-populations.pdf Date last updated: May 21, 2014.

6 Fattorini L, Migliori GB, Cassone A, et al. Proficiency testing of first- and second-line anti-tuberculosis drugs in Italy. Eur Respir J 2012; 39: 1263-1266.

7 World Health Organization. Global Tuberculosis Report 2014. WHO/HTM/TB/2014.08. Geneva, WHO, 2014

8 Lukoye D, Ssengooba W, Musisi K, et al. Variation and risk factors of drug resistant tuberculosis in sub-Saharan Africa: a systematic review and meta-analysis. BMC Public Health 2015; 15: 291.

9 Migliori GB, Dheda K, Centis R, et al. Review of multidrug-resistant and extensively drug-resistant TB: global perspectives with a focus on sub-Saharan Africa. Trop Med Int Health 2010; 15: 1052-1066.

10 Ben Amor Y, Nemser B, Singh A, et al. Underreported threat of multidrug-resistant tuberculosis in Africa. Emerg Infect Dis 2008; 14: 1345-1352.

11 Kidenya BR, Webster LE, Behan S, et al. Epidemiology and genetic diversity of multidrug-resistant tuberculosis in East Africa. Tuberculosis (Edinb) 2014; 94: 1-7.

12 Jabeen K, Shakoor S, Hasan R. Fluoroquinolone-resistant tuberculosis: implications in settings with weak healthcare systems. Int J Infect Dis 2015; 32: 118-123.

13 Cegielski JP, Dalton T, Yagui M, et al. Extensive drug resistance acquired during treatment of multidrug-resistant tuberculosis. Clin Infect Dis 2014; 59: 1049-1063.

14 D'Ambrosio L, Dara M, Tadolini M, et al. Tuberculosis elimination: theory and practice in Europe. Eur Respir J 2014; 43: 1410-1420.

15 Lönnroth K, Migliori GB, Abubakar I, et al. Towards tuberculosis elimination: an action framework for low-incidence countries. Eur Respir J 2015; 45: 928-952. 